\title{
Cosmological Evolution of Interacting Dark Energy Models with Mass Varying Neutrinos
}

\author{
Xiao-Jun Bi \\ Key laboratory of particle astrophysics, Institute of High Energy Physics, \\ Chinese Academy of Sciences, P.O. Box 918-3, Beijing 100049, People's Republic of China \\ Bo Feng, Hong Li, and Xinmin Zhang \\ Theoretical Physics Division, Institute of High Energy Physics, \\ Chinese Academy of Sciences, P.O. Box 918-4, Beijing 100049, People's Republic of China
}

\begin{abstract}
In this paper we consider the cosmological implications of dark energy models with a coupled system of a dynamical scalar field (the quintessence) and the neutrinos. By detailed numerical calculations we study the various possibilities on the evolution and the fates of the universe in this class of models. Our results show that due to the interaction with quintessence, neutrinos could be dominant over the quintessence in the future universe, however would eventually decay away.
\end{abstract}

\section{INTRODUCTION}

Recent data from type Ia supernova (SN Ia) 1] and cosmic microwave background $(\mathrm{CMB})$ radiation [2] have provided strong evidences for a spatially flat and accelerating universe at the present time. In the context of Friedmann-Robertson-Walker cosmology, this acceleration is attributed to the domination of a new matter component with negative pressure, dubbed dark energy [3]. The simplest candidate for dark energy seems to be a remnant small cosmological constant or vacuum energy with $\rho \sim\left(2 \times 10^{-3} \mathrm{eV}\right)^{4}$. This energy scale $\sim 10^{-3} \mathrm{eV}$ is smaller than the known energy scales in particle physics except that of the neutrino masses, which is comparable to the scale of dark energy.

The dark energy could also be due to a dynamical component, such as a canonical scalar field $\phi$, named quintessence [4, 5, 6]. Cosmological observations indicate that the potential of the quintessence field should be very flat around the present epoch. Consequently the effective mass should be extremely small, $m_{Q} \sim 10^{-33}$ $\mathrm{eV}$, which surprisingly is also connected to the neutrino masses via a see-saw formula $m_{Q} \sim m_{\nu}^{2} / m_{p l}$, with $m_{p l}$ being the Planck mass.

Are there any connections between the neutrinos and dark energy? Given the arguments above it is quite interesting to make such a speculation on this connection. If yes, in terms of the language of particle physics, it requires the existence of new dynamics and new interactions between the neutrino and the dark energy sector. Recently there are quite a few studies in the literature on the possible realization of the models connecting neutrinos and dark energy ${ }^{1}$ 7, $\left.8,19,10,11,12,13,14,15,16\right]$.

\footnotetext{
${ }^{1}$ In analog to the idea of top quark condensate as a mechanism of generating the electroweak scale, one might think that the neutrino condensate gives rise to the scale of dark energy. In this sense the dark energy scalar field, such as quintessence behaves like a bound state of the neutrinos. Effectively the system of
}

Qualitatively these models have made at least two interesting predictions: 1) neutrino masses are not constant, but vary during the evolution of the universe; 2) CPT is violated in the neutrino sector due to the $\mathrm{CPT}$ violating Ether during the evolution of the quintessence scalar field [14]. Quantitatively these predictions will depend on the dynamics governing the coupled system of the neutrinos and dark energy.

One of the possible couplings between the neutrinos and the scalar field is the derivative interaction 14]:

$$
\mathcal{L} \sim \frac{\partial_{\mu} \phi}{\Lambda} \bar{\nu}_{L} \gamma^{\mu} \nu_{L}
$$

During the evolution of a homogeneous quintessence scalar field, $\dot{\phi}$ does not vanish and gives rise to CPT violation in the neutrino sector. However, since $\dot{\phi}$ is very small at the present epoch this type of cosmological CPT violation is predicted to be much smaller than the current experimental limits. But in the early universe with high temperature it has been shown in Ref. 14 that this CPT violation is large enough for the generation of the baryon number asymmetry via leptogenesis. This new mechanism 14, 17] for baryogenesis/leptogenesis provides a unified picture for dark energy and baryon matter of the universe.

Another type of the interaction between the neutrinos and dark energy is that the quintessence field couples to the neutrino mass term. In the minimal extension of the standard model of particle physics, the neutrino masses can be described by a dimension- 5 operator

$$
\mathcal{L}_{\psi}=\frac{2}{f} l_{L} l_{L} H H+\text { h.c. },
$$

where $f$ is the scale of new physics beyond the standard model which generates the $B-L$ violation, $l_{L}, H$ are

dark energy sector consists of neutrinos and (single or multi) scalars which interact with each other given (approximately) by an effective Lagrangian similar to Eqs. 6) and (1). 
the left-handed lepton and Higgs doublets respectively. When the Higgs field gets a vacuum expectation value $<H>\sim v$, the left-handed neutrino receives a Majorana mass $m_{\nu} \sim \frac{v^{2}}{f}$. In Ref. [8] we considered an interaction between the neutrinos and the quintessence

$$
\mathcal{L}_{i n t}=\beta \frac{\phi}{M_{p l}} \frac{2}{f} l_{L} l_{L} H H+h . c,
$$

where $\beta$ is the coefficient characterizing the strength of the interaction between quintessence and the neutrinos. In this scenario the neutrino masses vary during the evolution of the universe and the neutrino mass limits imposed by the baryogenesis are modified.

The operator (2) is not renormalizable, which in principle can be generated by integrating out the heavy particles. For example, in the model of the minimal see-saw mechanism [18] for the neutrino masses, we have

$$
\mathcal{L}_{\text {neutrino }}=h_{i j} \bar{l}_{L i} N_{R j} H+\frac{1}{2} M_{i j} \bar{N}_{R i}^{c} N_{R j}+\text { h.c. },
$$

where $M_{i j}$ is the mass matrix of the right-handed neutrinos and the Dirac mass of neutrinos is given by $m_{D} \equiv$ $h_{i j}<H>$. Integrating out the heavy right-handed neutrinos will generate the operator in (21). However, as pointed out in Ref. [8], to make the light neutrino masses vary there are various possibilities, such as by coupling the quintessence field to the Dirac masses and/or the Majorana masses of the right-handed neutrinos. In Ref. 10] we have proposed a model of mass varying righthanded neutrinos. In this model the right-handed neutrino masses $M_{i}$ are assumed to be a function of the quintessence field $M_{i}(\phi)=\bar{M}_{i} e^{\beta \frac{\phi}{M_{p l}}}$. Integrating out the right-handed neutrinos will generate a dimension-5 operator like in (3), with the light neutrino masses varying in the following way

$$
\mathcal{L}_{\not}=e^{-\beta \frac{\phi}{M_{p l}}} \frac{2}{f} l_{L} l_{L} H H+\text { h.c. } .
$$

In this paper we will give a systematic study on the effects for a coupled system of light neutrinos and the quintessence field, especially on the late-time evolution and the fate of the universe. We will show numerically that 1) unlike the case in the absence of the interaction the relic neutrino energy density $\Omega_{\nu}$ may not decrease, instead it can track the energy density of the quintessence field. In the future, all of the pressureless matter may be diluted away and only the components of the neutrinos and quintessence remain. 2) Neutrino masses will increase as the universe expands, however when kinematically allowed the neutrinos will decay for instance into electrons and pion mesons. Since the electrons and the mesons are not coupled to the dark sector, the fate of the universe is governed by the "pure" dynamics of the quintessence field. In this paper we will quantify the moment when the neutrinos decay by the numerical calculation.
The paper is organized as follows: in Section II we will study the general features of the dark energy models with mass varying neutrinos and discuss analytically the behavior of the evolution of the system. In Section III we present the numerical results for some specific models. Section IV gives discussions and conclusions.

\section{DARK ENERGY MODELS WITH MASS VARYING NEUTRINOS}

In this section we will study in detail the dark energy models where the neutrinos and the quintessence interact with each other. Specifically we start with a class of models given by the following Lagrangian

$$
\mathcal{L}=\mathcal{L}_{\nu}+\mathcal{L}_{\phi}+M(\phi) \bar{\nu} \nu,
$$

where $\mathcal{L}_{\nu}=\bar{\nu} i \not \partial \nu$ and $\mathcal{L}_{\phi}=\frac{1}{2} \partial_{\mu} \phi \partial^{\mu} \phi-V(\phi)$ with $V(\phi)$ being the quintessence potential; $M(\phi)$ being the $\phi$-dependent neutrino mass such as in Eqs. (3) and (5). In this paper we consider mainly the models of neutrino coupling to the quintessence via the mass term.

As shown in Eq.(6) different dark energy models are specified by the form of $V(\phi)$ and $M(\phi)$. In this paper we will consider two classes of models, one has the exponential form of $V(\phi)$ and $M(\phi)$ and the other has the power law form. In this section we will give a general analysis of the behavior of the system and its condition for the attractor solution.

The equation of motion for the scalar field $\phi$ is given by

$$
\ddot{\phi}+3 H \dot{\phi}+\frac{d V}{d \phi}+\frac{d V_{I}}{d \phi}=0
$$

where

$$
\frac{d V_{I}}{d \phi}=\frac{d M}{d \phi} n\left\langle\frac{M}{E}\right\rangle
$$

is the source term by the interaction between the neutrinos and the scalar field, with $n$ and $E$ being the number density and energy of the neutrinos respectively, \langle\rangle indicates the thermal average. For relativistic neutrinos, the term $\frac{d V_{I}}{d \phi}$ is greatly suppressed and the neutrinos decouple from quintessence. For non-relativistic neutrinos, the effective potential of the system is given by $V_{\text {eff }}(\phi)=V(\phi)+n M(\phi)$.

For this coupled system, the energy for each component does not conserve. It is easy to get that the energy density of neutrinos evolutes as

$$
\dot{\rho}_{\nu}+3 H \rho_{\nu}=n M^{\prime} \dot{\phi},
$$

where $M^{\prime}=d M / d \phi$. Correspondingly due to the conservation of energy-momentum tensor of the whole system, the fluid equation of the scalar field $\phi$ is

$$
\dot{\rho}_{\phi}+3 H \rho_{\phi}\left(1+w_{\phi}\right)=-n M^{\prime} \dot{\phi},
$$


where $w_{\phi}=\frac{p_{\phi}}{\rho_{\phi}}=\frac{\frac{1}{2} \dot{\phi}^{2}-V(\phi)}{\frac{1}{2} \dot{\phi}^{2}+V(\phi)}$ is the equation of state of the scalar field. We can define the effective equation of states $w_{\nu}^{e f f}$ and $w_{\phi}^{e f f}$ as

$$
\begin{gathered}
\dot{\rho}_{\nu}+3 H \rho_{\nu}\left(1+w_{\nu}^{e f f}\right)=0, \\
\dot{\rho}_{\phi}+3 H \rho_{\phi}\left(1+w_{\phi}^{e f f}\right)=0,
\end{gathered}
$$

where

$$
w_{\nu}^{e f f}=-\frac{n M^{\prime} \dot{\phi}}{3 H \rho_{\nu}}=-\frac{1}{3} \frac{\partial \log M}{\partial \phi} \phi^{\prime}
$$

and

$$
w_{\phi}^{e f f}=w_{\phi}+\frac{1}{3} \frac{\partial \log M}{\partial \phi} \phi^{\prime} \frac{\rho_{\nu}}{\rho_{\phi}}
$$

with $\phi^{\prime}=\frac{\partial \phi}{\partial \log a}=\frac{\dot{\phi}}{H}$.

The effective equation of states $w_{\nu}^{e f f}$ and $w_{\phi}^{e f f}$ describe the actual rate of the energy density decrease of the two components respectively as the universe expands. When the two equation of states become equal we expect the two components to remain a constant ratio in energy densities. In the future when the energy densities of all other components of the universe dilute away and requiring $w_{\phi}^{e f f}=w_{\nu}^{e f f}$, we have

$$
\Omega_{\phi}=\frac{\phi^{\prime 2}}{3}+\frac{1}{3} \frac{\partial \log M}{\partial \phi} \phi^{\prime},
$$

where we have set $8 \pi G=1$ and used the relation $\Omega_{\phi}=$ $\frac{\phi^{\prime 2}}{6}+\Omega_{V}$. We can use the requirement of a constant ratio of the energy densities to derive the attractor solution of the system.

We first consider the class of models with exponential forms of $V(\phi)$ and $M(\phi)$, which have an attractor solution and will be shown below. For $M(\phi)=\bar{M} e^{-\lambda \phi}$ we have $\frac{\partial \log M}{\partial \phi}=-\lambda$. Requiring

$$
\frac{d \Omega_{\phi}}{d \log a}=0
$$

we get

$$
\left(2 \phi^{\prime}-\lambda\right) \phi^{\prime \prime}=0 .
$$

This leads to the solutions $\phi^{\prime}=\lambda / 2$ or $\phi^{\prime \prime}=0$. The first solution gives $\Omega_{\phi}<0$. For $\phi^{\prime \prime}=0$, we have $\phi=$ $\phi_{0}+\lambda^{\prime} \log a$. This solution leads to

$$
\Omega_{\phi}=\frac{1}{3}\left(\lambda^{\prime 2}-\lambda \lambda^{\prime}\right) .
$$

For the exponential potential $V=V_{0} e^{\beta \phi}$, we have the equation of motion [19]

$$
\frac{\rho_{m}+\rho_{\gamma}+\rho_{\nu}+V}{1-\phi^{\prime 2} / 6} \frac{\phi^{\prime \prime}}{3}+\left(\rho_{m}+\frac{2}{3} \rho_{\gamma}+2 V\right) \frac{\phi^{\prime}}{2}=-\beta V+\lambda \rho_{\nu} .
$$

From Eq. (19) we can get

$$
\Omega_{\phi}=\frac{\beta \frac{\lambda^{\prime 2}}{6}+\lambda-\frac{\lambda^{\prime}}{2}+\frac{\lambda^{\prime 3}}{6}}{\frac{\lambda^{\prime}}{2}+\beta+\lambda} .
$$

Eqs. (18) and (20) give the unique solution:

$$
\lambda^{\prime}=\frac{-3}{\lambda+\beta} .
$$

This is the attractor solution given in Ref. 19 in the discussions of a coupled system of quintessence and dark matter.

The exponential potential has very good attractor behavior and generically the coupled system is very close to the attractor region today and leads to a constant ratio of the two coupled components' energy densities. This scenario seems to be less interesting to us, since the evolution of the universe is still dominated by the dynamics of the scalar field with a negligible neutrino component.

In the following we will consider a class of power law models where $M=\bar{M} \phi^{-\beta}$ and $V(\phi)=V_{0} \phi^{\alpha}, \beta$ and $\alpha$ are the two model parameters to be specified for the numerical calculations in the next section. In this class of models, $\frac{\partial \log M}{\partial \phi}=-\frac{\beta}{\phi}$ and we have

$$
\Omega_{\phi}=\frac{\phi^{\prime 2}}{3}-\frac{\beta \phi^{\prime}}{3 \phi} .
$$

Demanding that $\Omega_{\phi}$ remains a constant during the evolution of the universe, one gets

$$
2 \phi^{2} \phi^{\prime} \phi^{\prime \prime}-\beta \phi \phi^{\prime \prime}+\beta \phi^{2}=0 .
$$

In the next section we will present the detailed numerical results on the evolutions of the universe. Specifically we will focus on the power law models and on the behavior of the solution to the equation above, when $\phi$ tends to zero and $\phi^{\prime} / \phi$ tends to a constant. Our results will show explicitly the importance of the neutrino component and the interaction with quintessence in determining the evolution and the fate of the universe.

\section{NUMERICAL RESULTS}

In this section, with the power law forms of interaction and potential, we will present our numerical results for the evolution of the coupled system given in Eq. (6). We will show that this class of models can satisfy the observations within the model parameter space, furthermore predict different fates of the universe. At high temperatures, the neutrinos are relativistic and decoupled from the scalar field, as shown in Eq. (8). At low temperatures, the neutrinos begin to affect the evolutions of the scalar field. Specifically we notice that the system will undergo a period of oscillation before entering the attractor region. In the period of oscillation, the equation 


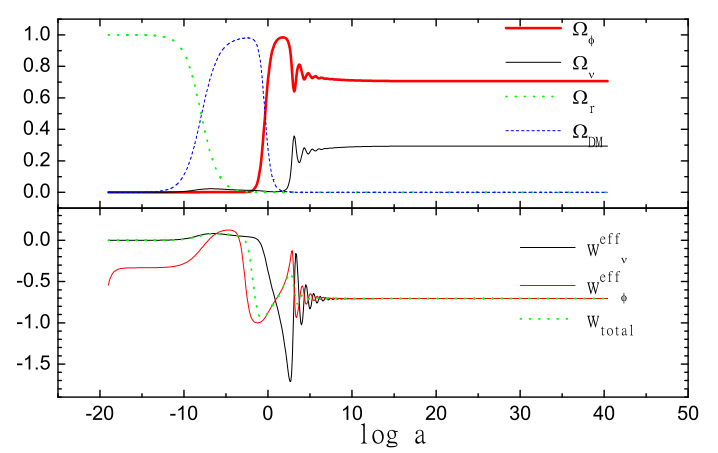

FIG. 1: The $\Omega_{i}=\frac{\rho_{i}}{\rho_{c}}$ and effective equation of state $W$ as functions of the scale factor $\log a$ for $V=V_{0} \phi^{5}$ and $M=$ $\bar{M} \phi^{-12}$.

of state of neutrino can be below -1, so its energy density can grow up and become comparable with the scalar field or dark matter. Therefore, the fate of the universe as we show below can be quite different from the dark energy model in the absence of the interaction with neutrinos. When neutrinos do not interact with dark energy, the density ratio between dark matter and neutrinos remains constant and typically dark energy will dominant the late time universe. However as shown below, the universe can be dominant with dark energy and neutrinos after introducing the interaction, with constant ratio of the energy densities of the two components. On the other hand, the universe can also be dominated by neutrinos and dark matter with negligible dark energy in another scenario. In the numerical study we have taken the parameter values today as $h \approx 0.7, \Omega_{\phi} \approx 0.7, \Omega_{m} \approx 0.3$ and $\Omega_{\nu}<0.02$.

In Fig. 11 by specifying the potential $V=V_{0} \phi^{5}$ and the neutrino mass $M=\bar{M} \phi^{-12}$, we plot the evolution of $\Omega_{i}=\frac{\rho_{i}}{\rho_{c}}$ and the effective equation of states as function of the scale factor $\log a$. The effective equation of states in the figure are defined in Eqs. (13) and (14). $w_{\text {total }}=$ $P_{\text {total }} / \rho_{\text {total }}$ here is the equation of state of the whole system. After a short period of oscillation, the energy density fraction of the scalar field decreases while the component of neutrino increases to around $30 \%$. The universe will be dominated by the two components in the future and the ratio of the two components keeps constant for ever. This picture corresponds to a solution of the forementioned equation (23) when $\phi$ approaches zero and $\phi^{\prime} / \phi$ remains nonvanishing. ${ }^{2}$

In the case of interacting neutrino and dark energy

\footnotetext{
${ }^{2}$ For simplicity we have taken neutrinos to be matter like in the early epoch. This does not change our late time picture when considering the neutrino behavior at the early times.
}

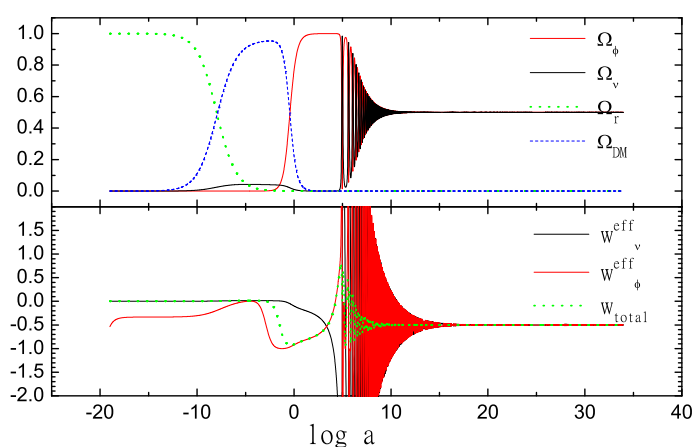

FIG. 2: The $\Omega_{i}$ and the effective equation of state $W$ as functions of the scale factor $\log a$ for $V=V_{0} \phi^{4}$ and $M=$ $\bar{M} \phi^{-4}$.

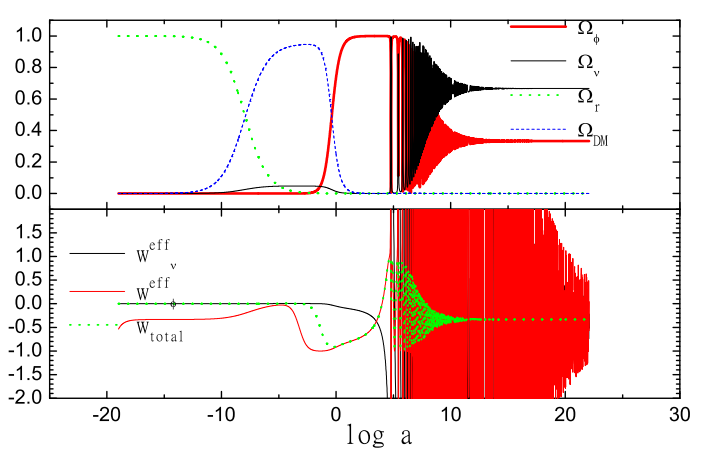

FIG. 3: The $\Omega$ and the effective equation of state $W$ as functions of the scale factor $\log a$ for $V=V_{0} \phi^{4}$ and $M=$ $\bar{M} \phi^{-2}$.

there is typically a period of "build-in" phase before the system arrives the late time stable states. Fig. 11 stands for the case where the system enters quickly the stable phase. We find that to achieve a universe with a larger fraction of neutrinos, it will typically take a considerably longer time for the "build-in" phase. As an example we consider a model with $V=V_{0} \phi^{4}$ and the neutrino mass $M=\bar{M} \phi^{-4}$. In Fig. 2 we plot the evolution of $\Omega_{i}$ and the effective equation of states for this model. One can see after the period of oscillation, the universe approaches to a state consisting equally of the neutrino and the scalar field: $\Omega_{\nu}=\Omega_{\phi}=50 \%$. The equation of state of the system at the tracking region is $w_{\text {total }}=-0.5$. A more interesting case is plotted in Fig. 3] for $V=V_{0} \phi^{4}$ and $M=\bar{M} \phi^{-2}$. The universe will finally be dominated by the neutrinos with $\Omega_{\nu} \approx 2 / 3$ while $\Omega_{\phi} \approx 1 / 3$. The equation of state of the system is $w_{\text {total }} \approx-1 / 3$.

The oscillating phase is determined by two conditions. One is the asymptotic behavior of $V_{e f f}^{\prime}$. In the case of a power law quintessence potential and neutrino masses, 


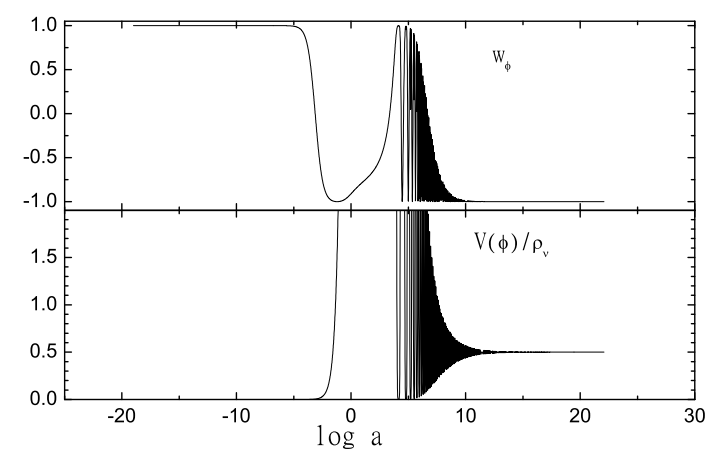

FIG. 4: $w_{\phi}$ and the ratio of $V(\phi)$ with neutrino energy density as functions of the scale factor $\log a$ for $V=V_{0} \phi^{4}$ and $M=$ $\bar{M} \phi^{-2}$.

$V_{\text {eff }}^{\prime}$ gets asymptotically to zero during the evolution. The other condition is the magnitude of $V^{\prime \prime}$ compared with the Hubble expansion rate. The scalar field starts to oscillate at the time $V_{e f f}^{\prime \prime} \sim H^{2}$. Due to the Hubble friction the oscillation damps with time. When the oscillating amplitude is small enough the kinetic term of $\phi$ becomes negligible, i.e. $\frac{1}{2} \dot{\phi}^{2} \ll V(\phi)$, and $w_{\phi}$ tends to -1 . For the power law form we will then have $\alpha V(\phi) \simeq \beta \rho_{\nu}$, $w_{\text {total }}=\frac{\frac{1}{2} \dot{\phi}^{2}-V(\phi)}{\frac{1}{2} \dot{\phi}^{2}+V(\phi)+\rho_{\nu}} \simeq \frac{-V(\phi)}{V(\phi)+\rho_{\nu}}=\frac{-\beta}{\alpha+\beta}$ and $\Omega_{\phi} \simeq \frac{\beta}{\alpha+\beta}$. Taking the model in Fig. 3] as an example, we delineate in Fig. 4 the corresponding $w_{\phi}$ and $V(\phi) / \rho_{\nu}$ as functions of the scale factor $\log a$. The contribution of the kinetic term oscillates and decreases with time. After the freezing of $w_{\phi}=-1$, the ratio $V(\phi) / \rho_{\nu}$ approaches $1 / 2$ and $V_{e f f}^{\prime} \sim 0$. Comparing with Fig. 3. we notice that $w_{\text {total }}$ and $\Omega_{\phi}$ become nearly constant after $w_{\phi}$ approaches -1 . However, we should point out that the damped oscillation lasts for a longer time and $\Omega_{\phi}^{\prime} / \Omega_{\phi}$ is not yet negligible for $\log a>10$ in the shown range of $\log a$ in Fig. 3] and Fig. 4 Meanwhile the two effective equations of state remain damped oscillating around $w_{\text {total }}$.

As shown above in the class of models with the scalar potential $V(\phi)$ and the neutrino mass $M(\phi)$ being the power law form the neutrino masses keep increasing and, unlike the dark matter, its energy density does not dilute away. In some cases neutrinos will even dominate over the quintessence. These results, however depend on the couplings of the neutrinos to quintessence. As an example we take $V(\phi)=V_{0} \phi^{\alpha}$ while $M=\bar{M} \exp (-\beta \phi)$. The behavior of neutrinos and dark energy cannot remain symmetric and their ratio cannot take as constant during late time evolution. The dark energy will transfer its energy density to neutrinos before their decoupling. The universe becomes neutrino dominant with a constant ratio between $\Omega_{\phi}$ and $\Omega_{m}$. In Fig. 5]we realize this picture with $V(\phi)=V_{0} \phi^{4}$ and $M=\bar{M} \exp \left(-4 \phi / M_{P l}\right)$. The universe evolves approaching a state dominated by matter and neutrino, where the effective equation of state $w_{\text {total }}$

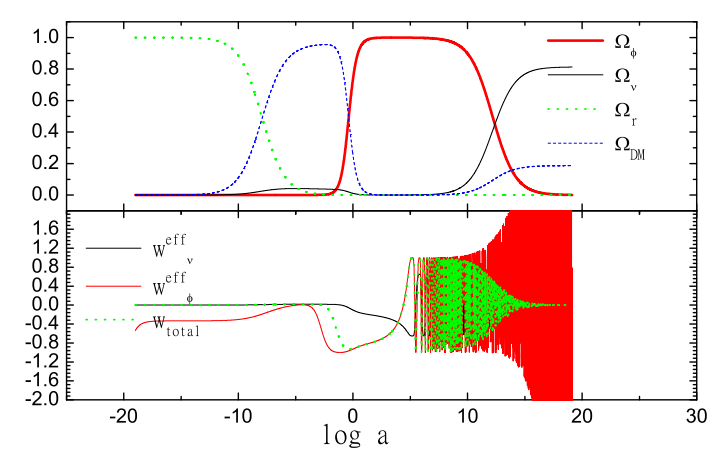

FIG. 5: The $\Omega$ and the effective equation of state $W$ as functions of the scale factor $\log a$ for $V=V_{0} \phi^{4}$ and $M=$ $\bar{M} e^{-4 \phi / M_{P l}}$.

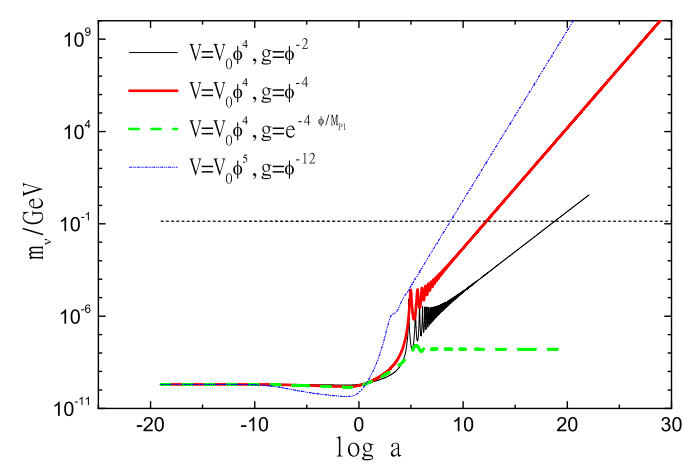

FIG. 6: The neutrino mass as functions of the scale factor $\log a$, where $g=M(\phi) / \bar{M}$.

is zero. Due to the different behavior of $V_{e f f}^{\prime}$ from the previous power law cases, the neutrino mass in Fig. [5 will become constant in the future when the universe stops acceleration.

Generally speaking the mass of neutrinos will vary as the evolution of the coupled system. It is easy to show that in general $M(\phi)=\bar{M} a^{-3 w_{\nu}^{e f f}}$ with $a$ being the scale factor. For $w_{\nu}^{e f f}<0$, the neutrinos are lighter in the past, but become heavier in the future. In Fig. 6] we plot the neutrino mass as a function of the scale factor $\log a$ for the models discussed in this section. It should be noticed that the neutrino mass will increase forever as the expansion of the universe for the power law cases. When the neutrinos are heavy enough, however they will decay to electron and pion $\nu \rightarrow e^{ \pm}+\pi^{\mp}$. The life time of the decay is given by

$$
\tau_{\nu}=6 . \times 10^{-9} \mathrm{sec} \cdot\left(1-\frac{m_{\pi}^{2}}{m_{\nu}^{2}}\right)^{-2} .
$$

Therefore, the neutrinos will decay away immediately 
once they cross the energy threshold. The energy density of neutrinos will then be transferred to matter, which may eventually be diluted away with the expansion of the universe. In this case the scalar field will finally dominate the universe and determine the evolution of the universe. In Fig. 6] for an intuitive view, the moment when the decay occurs is set as $m_{\nu}=m_{\pi}+m_{e}$.

\section{DISCUSSIONS AND CONCLUSIONS}

In this paper we have studied the dynamics of the coupled system described by Eq. (6). We paid particular attention to the effects of the back reaction of the thermal neutrino bath in the universe to the evolution of the scalar field - the quintessence - due to the $\phi$-dependent mass term [20].

The coupled system has many interesting phenomenologies. First of all, during the evolution of the universe the neutrino masses vary. This effect will make the matter power spectrum observed from the Large Scale Structure(LSS) survey different from that for a constant neutrino mass. Consequently the present cosmological limits on the neutrino mass $m_{i}<0.6 \mathrm{eV}$ will be relaxed in the scenario with mass varying neutrinos [21]. In the current paper we mainly focus on the models where neutrinos can track dark energy. Although the mass of neutrinos varies significantly around the tracking regime, it has changed very little around today. This is due to the fact that the coupled system of neutrinos and dark energy has been stringently constrained by the current observations. It is trivial if the system has entered the tracking regime today when the fraction of the neutrino energy density is negligible 22. Meanwhile as the tracking regime has been kept in the far future from today, the mass of neutrinos has changed little till the present epoch due to the slow rolling of $\phi$. In this sense our scenario is hard to distinguish experimentally from the standard case with constant neutrino masses. On the other hand, however, if we do not expect a neutrino dominant future universe and the present epoch is closer to the tracking regime, neutrino mass could have evolved from the past to now, as shown in the example of $V=V_{0} \phi^{5}$ and $M=\bar{M} \phi^{-12}$ in Fig. [6] We could then expect some distinguishable imprints from the uncoupled case 21].

In addition, if the neutrino mass is produced through the seesaw mechanism and the scalar field only couples with the right-handed neutrinos, this scenario will change the predictions of the leptogenesis mechanism. As shown

3 For the phantom scalar field, the effective Lagrangian after including the quantum effects from the derivative couplings is given by

$$
\mathcal{L}_{e f f}=\frac{1}{2}\left(-1+\frac{T^{2}}{12 \Lambda^{2}}\right) \partial_{\mu} \phi \partial^{\mu} \phi .
$$

explicitly in Ref. [10] the reheating temperature required by leptogenesis can be lowered and the bound on the light neutrino mass set by leptogenesis can also be relaxed.

Finally, the derivative coupling in Eq. (1) contributes also to the kinetic term. It is easy to show that the quantum effect at zero temperature vanishes. However, at finite temperature $T$ the corrections are given by

$$
\mathcal{L}_{\text {correct }}^{T}=\frac{1}{2}\left(\frac{T^{2}}{12 \Lambda^{2}}\right) \partial_{\mu} \phi \partial^{\mu} \phi .
$$

The effect is negligible at low temperature $T \ll \Lambda$. However, it could be important at very high temperatures when $T \sim \Lambda$. Ref. 14] has also considered another type of the back reaction caused by the derivative couplings in Eq. (11) and shown explicitly the negligible effects at low temperature ${ }^{3}$.

We should point out that the main purpose of the current paper is the phenomenological study of the coupled system of dark energy and neutrinos. We do not try to build the dynamical relation of neutrinos and dark energy and a possibility of neutrino condensate to achieve dark energy is pointed out in the footnote 1. Attempts for a possible dynamical link between the values of the neutrino mass and dark energy today have been studied in Refs. 5. 24]. Furthermore as shown in the previous studies of tracking dark energy models one usually needs some fine tuning for the energy density of quintessence today [6].

In summary we have presented a systematic study on the cosmological evolution of the dark energy model with a coupled system including a dynamical scalar field (the quintessence) and the neutrinos. We have shown that the dynamics of this system drives the universe accelerating at present and leads to various interesting possibilities on the evolution of the universe. In our models, the neutrinos may dominate the universe in the future. However, in most scenarios, the neutrinos will eventually decay away.

\section{Acknowledgments}

We thank Peihong Gu, Roberto Peccei, Yun-Song Piao and Bing-Lin Young for discussions. This work is supported in part by the Natural Science Foundation of China under the Grant Nos. 10575111, 10105004, 10120130794, 90303004, 19925523 and by the Ministry of Science and Technology of China under the Grant No. NKBRSF G19990754.

At high temperatures the system is a canonical scalar field while at low temperature a 'phase transition' occurs and the system turns to the phase of phantom. This transition is somewhat favored by the present SN Ia observations [23]. 
arXiv: astro-ph/0302207 D. N. Spergel et. al., Astrophys. J. Suppl. 148, 175 (2003), arXiv: astro-ph/030209.

[3] M.S. Turner, astro-ph/0108103

[4] B. Ratra and P.J.E. Peebles, Phys. Rev. D 37, 3406 (1988); C. Wetterich, Nucl. Phys. B 302, 302 (1988).

[5] J.A. Frieman, C.T. Hill, A. Stebbins, and I. Waga, Phys. Rev. Lett. 75, 2077 (1995).

[6] I. Zlatev, L. Wang, and P.J. Steinhardt, Phys. Rev. Lett. 82, 896 (1999); P.J. Steinhardt, L. Wang and I. Zlatev, Phys. Rev. D 59, 123504 (1999).

[7] P. Q. Hung, hep-ph/0010126 This paper is on the interaction between the quintessence and the sterile neutrinos.

[8] P. Gu, X. Wang and X. Zhang, Phys. Rev. D 68, 087301 (2003).

[9] R. Fardon, A. E. Nelson, N. Weiner, astro-ph/0309800

[10] X.-J. Bi, P. Gu, X. Wang, X. Zhang Phys. Rev. D 69, 113007 (2004).

[11] P. Q. Hung, H. Pas, astro-ph/0311131

[12] D. B. Kaplan, A. E. Nelson, N. Weiner, Phys. Rev. Lett. 93, 091801 (2004).

[13] R.D. Peccei, hep-ph/0404277 hep-ph/0411137

[14] M. Li, X. Wang, B. Feng and X. Zhang, Phys. Rev. D 65, 103511 (2002); M. Li and X. Zhang, hep-ph/0209093 Phys. Lett. B 573, 20 (2003).

[15] X. Zhang, hep-ph/0410292 H. Li, Z. Dai and X. Zhang, hep-ph/0411228

[16] E. I. Guendelman, A. B. Kaganovich, hep-th/0411188

[17] A. DeFelice, S. Nasri and M. Trodden, Phys. Rev. D 67, 043509 (2003); M. Yamaguchi, Phys. Rev. D 68, 063507 (2003); R. Brandenberger and M. Yamaguchi, Phys. Rev. D 68, 023505 (2003); M. Trodden, hep-ph/0302151 T. Chiba, F. Takahashi, and M. Yamaguchi, Phys. Rev.
Lett. 92, 011301 (2004); G.L. Alberghi, R. Casadio, and A. Tronconi, hep-ph/0310052

[18] M. Gell-Mann, P. Ramond and R. Slansky, Proceedings of the Supergravity Stony Brook Workshop, New York, 1979, eds. P. Van Nieuwenhuizen and D. Freedman (North-Holland, Amsterdam); T. Yanagida, Proceedings of the Workshop on Unified Theories and Baryon Number in the universe, Tsukuba, Japan 1979 (eds. A. Sawada and A. Sugamoto, KEK Report No. 79-18, Tsukuba); R. N. Mohapatra and G. Senjanovic, Phys. Rev. Lett. 44, 912 (1980); S. L. Glashow, Caraese lectures, (1979).

[19] D. Comelli, M. Pietroni, A. Riotto, Phys. Lett. B 571, 115 (2003); U. Franca and R. Rosenfeld, Phys. Rev. D 69, 063517 (2004).

[20] Gravitational backreaction to the Quintessence has been considered in: M. Li, W. Lin, X. Zhang and R. H. Brandenberger, Phys. Rev. D 65, 023519, (2002), hep-ph/0107160

[21] X. J. Bi, B. Feng, G. B. Zhao and X. Zhang, in prepration.

[22] It is also possible if neutino and quintessence have entered the tracking regime today, the observational constraints on the system are somewhat released compared with the uncoupled case. For a recent study on the coupled system see e.g. A.W. Brookfield, C. van de Bruck, D.F. Mota and D. Tocchini-Valentini, astro-ph/0503349

[23] e.g. U. Alam, V. Sahni, and A. A. Starobinsky, astro-ph/0403687 D. Huterer and A. Cooray, astro-ph/0404062 B. Feng, X. Wang and X. Zhang, astro-ph/0404224

[24] A. Singh, Phys. Rev. D 52, 6700 (1995). 